Service social

\title{
Pour réussir le recrutement des membres. L’importance des alliances avec la communauté
}

\section{Daniel Turcotte}

Volume 46, numéro 2-3, 1997

Groupes - Symposium 1997

URI : https://id.erudit.org/iderudit/706762ar

DOI : https://doi.org/10.7202/706762ar

Aller au sommaire du numéro

\section{Éditeur(s)}

École de service social de l'Université Laval

ISSN

1708-1734 (numérique)

Découvrir la revue

Citer cet article

Turcotte, D. (1997). Pour réussir le recrutement des membres. L'importance des alliances avec la communauté. Service social, 46(2-3), 101-126.

https://doi.org/10.7202/706762ar
Résumé de l'article

En service social, plusieurs interventions visent les personnes défavorisées et marginalisées généralement perçues comme réfractaires, voire hostiles, aux services sociaux. L'intervention de groupe constitue une méthode de choix pour intervenir auprès de ces personnes. En effet, le groupe offre un cadre favorable au développement des compétences et à l'apprentissage de nouveaux comportements. En outre, la force collective qui émerge d'un groupe contribue à atténuer les sentiments d'impuissance et de désespoir. Cependant, en raison de leur méfiance à l'égard des services, le recrutement des membres représente un défi de taille. Ce texte porte sur l'importance des alliances entre les différents intervenants. Après une brève révision des difficultés que pose le recrutement des personnes opprimées ou marginalisées, l'auteur propose un cadre pour analyser la décision d'accepter de l'aide et fournit quelques exemples de stratégies. 


\section{Pour réussir le recrutement des membres L'importance des alliances avec la communauté}

Daniel TURCOTTE

En service social, plusieurs interventions visent les personnes défavorisées et marginalisées généralement perçues comme réfractaires, voire hostiles, aux services sociaux. L'intervention de groupe constitue une méthode de choix pour intervenir auprès de ces personnes. En effet, le groupe offre un cadre favorable au développement des compétences et à l'apprentissage de nouveaux comportements. En outre, la force collective qui émerge d'un groupe contribue à atténuer les sentiments d'impuissance et de désespoir. Cependant, en raison de leur méfiance à l'égard des services, le recrutement des membres représente un défi de taille. Ce texte porte sur l'importance des alliances entre les différents intervenants. Après une brève révision des difficultés que pose le recrutement des personnes opprimées ou marginalisées, l'auteur propose un cadre pour analyser la décision d'accepter de l'aide et fournit quelques exemples de stratégies.

Marginalised and oppressed populations who are seen as reluctant to help are often served by social work services. Groupwork is a method of choice with these populations; group experience can reduce clients' denial and facilitate the development of more acceptable ways of dealing with their problems. Furthermore, the "strength in members" phenomenon in the group can increase their power and their control over their behaviour. However, recruiting members is usually very difficult among these populations. The present article emphasises the importance of partnership to develop effective strategies for recruitment. Some issues of practice with reluctant populations are reviewed, a model of help-seeking is proposed and some examples of strategies for recruiting members are presented. 


\section{INTRODUCTION}

En service social, une proportion grandissante des interventions vise les populations défavorisées, opprimées et marginalisées, ces populations dont les problèmes résultent d'une combinaison de facteurs personnels et structurels. Généralement perçues comme réfractaires, voire hostiles, aux services sociaux, ces personnes, qui sont généralement associées aux clientèles à risque, sont peu portées à demander de l'aide; elles rejettent même les offres de service les mieux intentionnées, provoquant ainsi chez les intervenants des sentiments d'incompétence et d'impuissance.

Plusieurs intervenants ne se sentent pas adéquatement préparés pour intervenir auprès de ces populations. Dans les faits, il existe peu de données théoriques pouvant guider l'intervention auprès des populations difficiles à rejoindre, ce qui conduit les intervenants à s'en reporter à leurs connaissances sur l'intervention auprès des clientèles volontaires. Or, ces repères se révèlent généralement inadéquats pour guider l'intervention auprès des populations défavorisées. Devant l'échec de leur action, les intervenants en viennent implicitement à " blâmer les clients " pour leur insuccès, situation qui entraîne, en contrepartie, une réaction où les clients blâment les intervenants pour leur inefficacité.

Le travail de groupe peut se révéler une méthode de choix pour intervenir auprès des personnes marginalisées et opprimées qui se tiennent à distance des services sociaux. En effet, en tant que milieu de partage et d'échange, le groupe offre un cadre favorable à l'apprentissage et au développement des compétences: "Small groups provide ideal conditions for group participants to experience, gain, exercise, and rehearse their "power" " (Hirayama et Hirayama, 1986). L'appartenance à un groupe facilite la reconnaissance de ses problèmes et l'identification de moyens pour changer sa réalité (Levine et Gallogly, 1985 cité dans Behroozi, 1992a); à travers le groupe, les membres apprennent à recevoir et à donner du feedback dans un contexte de soutien mutuel qui contribue à atténuer leur sentiment d'isolement. En outre, le groupe, avec ses mécanismes régulateurs, offre un cadre qui facilite l'apprentissage de nouveaux comportements. De plus, le fait de se sentir « tous dans le même bateau " permet aux membres de mieux composer avec leurs sentiments de honte et de culpabilité, contribuant ainsi à atténuer leurs 
réactions de défense, de résistance et de déni (Behroozi, 1992a). Le sentiment de force collective qui émerge d'un groupe contribue à augmenter le sentiment de contrôle sur sa vie, contrant alors les sentiments d'impuissance et de désespoir (Shulman, 1988). Ainsi, en utilisant le groupe comme contexte d'intervention, l'intervenant social peut contribuer à mettre en place un système d'aide au sein duquel les interactions entre les membres vont favoriser l'émergence de nouvelles modalités de perception, de pensée et d'action.

Si l'intervention de groupe est une méthode appropriée dans le cas des personnes difficiles à rejoindre, elle représente par ailleurs un défi de taille, notamment en ce qui a trait au recrutement. En effet, les efforts les plus louables d'analyse de besoins et de structuration de programme perdent tout leur sens si l'intervenant se bute à une absence de participants. Et même si un groupe vient répondre à des besoins largement reconnus, il n'est pas rare que des intervenants attendent pendant des semaines des personnes qu'on leur envoie et qui ne viennent jamais ou qu'ils se retrouvent avec un groupe composé de trois ou quatre personnes, alors qu'ils en espéraient une dizaine.

Le recrutement représente une étape cruciale du processus d'intervention puisque, pour qu'un groupe fonctionne de façon à favoriser l'émergence de l'aide mutuelle, non seulement il est nécessaire de rejoindre un nombre suffisant de personnes, mais il faut que ces personnes possèdent les caractéristiques recherchées. Le fait de rejoindre des personnes différentes de celles prévues peut conduire à une hétérogénéité qui rend très difficile la satisfaction des besoins de tous les participants, tout en comportant l'exigence d'ajuster les objectifs et le programme d'intervention.

Ce texte porte sur le recrutement des membres au sein des populations difficiles à rejoindre; il vise à souligner l'importance des alliances entre les différents intervenants œuvrant dans une communauté pour entrer en contact avec ces populations. II se divise en trois parties. La première touche les difficultés que pose le recrutement des personnes opprimées ou marginalisées en intervention de groupe. La seconde propose un cadre pour analyser la décision des personnes en difficulté de solliciter ou d'accepter de l'aide. La dernière relate des stratégies d'action qui peuvent être utilisées pour procéder au recrutement des membres. La structure de ce texte 
présente en alternance des données tirées de la littérature sur le sujet et des observations empiriques résultant d'une recherche sur des projets réalisés dans le cadre du Programme d'action communautaire pour les enfants ${ }^{1}$.

\section{LE RECRUTEMENT AU SEIN DES POPULATIONS DÉFAVORISÉES}

Pour les intervenants qui sont prêts à prendre le risque de travailler avec les populations défavorisées, l'intervention de groupe constitue une méthode particulièrement intéressante. Recevoir de l'aide dans un contexte de groupe peut se présenter comme une solution alternative attrayante pour des personnes qui sont réfractaires à l'idée de s'engager dans un processus de «thérapie ", puisque le groupe constitue un contexte moins intime que la relation dyadique professionnel-client; en ce sens, il convient davantage aux personnes réservées ou méfiantes (Shapiro,1984). Mais le groupe comporte aussi certains inconvénients : la confidentialité est plus difficile à préserver, la personne peut se sentir exposée à la réaction des autres membres ou elle peut être gênée par leurs attitudes, leurs réactions et leurs façons d'être. Cette méthode n'élimine donc pas la difficulté que posent la méfiance et l'insécurité face aux services sociaux.

Dans une recherche menée récemment auprès d'organismes communautaires réalisant des activités auprès de familles défavorisées, plusieurs intervenants ont souligné la complexité de recrutement parmi cette population. Certains motifs avancés par ces intervenants pour expliquer leurs difficultés de recrutement concernent la réticence des familles à risque à demander et à recevoir de l'aide, soit parce qu'elles ne se sentent pas à l'aise de se faire aider, comme c'est souvent le cas pour les membres de certaines communautés ethniques, soit parce qu'elles craignent d'en subir des conséquences négatives. Le tableau 1 illustre les deux principaux motifs mentionnés

1. Cette recherche a été réalisée par D. Turcotte, C. Samson, G. Lessard et A. Beaudoin dans le cadre d'un projet subventionné par Santé Canada. Les personnes intéressées à en connaître davantage sur la méthodologie et les résultats de cette recherche peuvent consulter le rapport produit par Turcotte, Samson, Lessard et Beaudoin (1997). 
par les intervenants, par rapport à la population visée, en explication à leurs difficultés de recrutement².

\section{Tableau 1}

\section{Les difficultés de recrutement attribuables à la population visée}

Réticence à demander de l'aide

Crainte des conséquences négatives
Il y a un autre problème [...] c'est de rejoindre réellement la clientèle cible. [...] Les gens qui sont les plus ciblés, on a de la misère à les faire sortir. $\left(n^{\circ} 2\right)$

Ce sont des gens difficiles à aborder, des gens qui ne demandent pas d'aide. II faut beaucoup de stimulation pour leur dire vous avez besoin d'aide. $\left(n^{\circ} 15\right)$

Ce que je trouve difficile c'est de faire tomber la méfiance des familles face aux services que l'on offre. Cette situation est particulièrement vraie pour les familles référées par la DPJ. Jusqu'à présent, les familles ne m'ont jamais rappelé. $\left(n^{\circ} 19\right)$

Elle n'a pas voulu parce que selon elle le projet est chapeauté par le CLSC et elle a peur que, si elle fait affaire avec eux, ils vont lui enlever ses enfants. $\left(n^{\circ} 8\right)$

Les explications données par les intervenants rejoignent plusieurs éléments mentionnés dans la littérature en ce qui a trait aux facteurs qui influencent la recherche d'aide. Ces éléments seront brièvement passés en revue.

\section{LES FACTEURS QUI INFLUENCENT LA RECHERCHE D'AIDE}

Les travaux sur la recherche d'aide ont conduit à identifier plusieurs facteurs qui exercent une influence sur la propension à demander ou à recevoir de l'aide. Gross et McMullen (1983) décomposent le processus qui conduit à une demande d'aide en trois étapes : 1) la perception d'une situation comme un problème pouvant profiter d'une aide extérieure; 2) la décision d'accepter le problème, de le régler seul ou de solliciter de l'aide et 3) la mise en place de stratégies permettant d'obtenir l'aide désirée. Ces étapes ne sont pas nécessairement linéaires, mais elles correspondent à une démarche où

2. Ce tableau est tiré du rapport de recherche Turcotte, Samson, Lessard, Beaudoin (1997). Les chiffres en fin de citation correspondent à un numéro attribué aléatoirement aux projets étudiés. 
l'action s'accompagne d'opérations cognitives et décisionnelles. Pour Rogler et Cotes (1993), le concept de " trajectoire ", défini comme la séquence de contacts qu'une personne en détresse établit avec des individus et des organisations pour obtenir de l'aide, traduit bien le caractère structuré de cette démarche. Différents facteurs peuvent influencer le cheminement de la personne dans cette trajectoire (voir également Cantin, 1998; Dulac, 1997; Turcotte, Damant, Lindsay, 1995). Globalement, il est possible de dégager deux grandes perspectives au regard des études qui portent sur la recherche d'aide. La première aborde l'étude des facteurs en se centrant sur les caractéristiques individuelles et les variables de l'environnement; la seconde s'intéresse aux interactions qui ont cours entre une personne en difficulté et un organisme qui propose des services.

\section{Les facteurs liés aux caractéristiques individuelles}

Plusieurs recherches empiriques indiquent que des caractéristiques comme le genre, la classe sociale et l'appartenance culturelle sont associées à la fréquence d'utilisation des services d'aide. L'écart entre les hommes et les femmes au niveau des diagnostics et des traitements en santé mentale est un phénomène bien connu (Johnson, 1988; Tousignant, 1987; Fischer et coll.,1983; Veroff, Douvan et Kulka, 1981). Plusieurs études soulignent également que les personnes ayant un statut socio-économique plus faible sont moins susceptibles de faire appel à l'aide institutionnelle ou formelle (Williams et Williams, 1983). Par ailleurs, lorsque ces personnes font une telle demande, elles ont tendance à adopter un style didactique, c'est-à-dire à demander des instructions précises sur la façon de résoudre leur problème, alors que les personnes de milieu aisé privilégient plus souvent un style de négociation : l'aide demandée est vue comme un élément visant à faciliter leur démarche de résolution de problème (Asser, 1978). La variation de la propension à demander de l'aide selon l'appartenance culturelle et ethnique est également un phénomène documenté (Fortin et Legault, 1994; Ying et Miller, 1992), tout comme l'influence de caractéristiques individuelles comme l'estime de soi, le site de contrôle, la capacité de parler de soi et les croyances, attentes et attitudes à propos de l'utilité de recourir à une aide extérieure (Tijhuis et collab., 1990; Good, Dell et Mintz, 1989; Nadler, 1983). 
Au-delà des caractéristiques individuelles, certains facteurs liés au contexte de vie peuvent entrer en ligne de compte dans la décision de s'engager dans une démarche d'aide : les informations normatives, le jugement des autres, la comparaison sociale, les attitudes de l'entourage. En fait, certains environnements tendent non seulement à nier certaines catégories de problèmes, mais contribuent à les maintenir (Bilodeau, 1994).

Parallèlement aux explications basées sur l'influence des caractéristiques individuelles et des variables de l'environnement, il existe une autre perspective d'analyse de ce phénomène qui, sans nier l'importance des facteurs individuels et environnementaux, met l'accent sur le fait que les humains sont des êtres rationnels dont les comportements ne sont pas entièrement déterminés par leur milieu. Selon cette perspective, le comportement d'une personne peut être analysé comme une action entreprise en vue d'obtenir certains résultats ou d'éviter certaines conséquences. Les recherches réalisées selon cette deuxième perspective se sont principalement intéressées à étudier les conséquences liées au fait de demander ou de recevoir de l'aide.

\section{La recherche d'aide abordée dans une perspective interactionniste}

Les recherches associées à la perspective interactionniste portent sur l'analyse des enjeux que comporte le fait de demander ou de recevoir de l'aide. La recherche d'aide y est abordée comme un comportement susceptible d'apporter des bénéfices, mais qui comporte également certains coûts. Deux modèles sont à la base de ces études : la théorie de l'échange social et la théorie de l'obligation.

Selon la théorie de l'échange social, les interactions humaines sont une suite d'échanges qui comportent des coûts et des bénéfices. Comme chaque individu a intérêt à minimiser ses coûts et à maximiser ses bénéfices, la personne aura tendance à éviter les situations et les comportements coûteux et à rechercher les interactions qui lui procurent des bénéfices. Donc, les personnes seront davantage portées à rechercher de l'aide si leur analyse les amène à y voir plus d'avantages que de coûts; d'où l'importance de saisir comment une personne évalue les coûts et bénéfices associés au fait de demander ou de recevoir de l'aide (Antonucci et Jackson, 1990; Vaux, 1988). 
La théorie de l'obligation apporte un éclairage complémentaire en avançant que le fait de recevoir un bénéfice crée un état d'obligation qui exige une forme de remboursement. Ainsi, les coûts de l'aide peuvent être d'autant plus significatifs que le fait de recevoir un bénéfice crée un sentiment d'obligation qui exige une forme de remboursement (Greenberg et Wescott, 1983).

Selon la perspective interactionniste, une conduite n'est jamais totalement prévisible, ni entièrement déterminée par les caractéristiques de la personne ou de l'environnement. Pour comprendre les comportements, il faut partir du point de vue de la personne et explorer l'analyse qu'elle fait de la situation dans laquelle elle se trouve; les objectifs qu'elle poursuit, les possibilités qui s'offrent à elle, les contraintes avec lesquelles elle doit composer. Dans cette exploration, il ne faut pas oublier que le fait de demander ou de recevoir de l'aide comporte généralement des coûts : perte d'autonomie, intrusion dans sa vie, pression au changement, réaction de l'entourage. Par exemple, Manor (1986; 1988) souligne qu'il existe chez toute personne une ambivalence entre le désir de partager avec d'autres et le besoin de préserver son autonomie; comme le fait de recevoir de l'aide implique une ouverture sur sa vie privée, l'aide peut être perçue comme une menace à l'autonomie. Dans le même sens, Breton (1991) relève trois motifs qui peuvent expliquer le fait que certaines personnes hésitent à demander de l'aide : 1) ces personnes craignent de subir un nouvel échec; 2) elles veulent écarter les risques de conséquences négatives que comporte le fait de recevoir de l'aide; ou 3) elles veulent maintenir le contrôle qu'elles exercent sur leur vie.

Si l'engagement dans une mesure d'aide exige au départ que la personne éprouve un sentiment d'inconfort ou d'insatisfaction devant sa réalité, cette impression n'est pas suffisante pour engager une action : la personne peut choisir de vivre avec cet inconfort ou tenter d'y remédier par elle-même. Par ailleurs, voir la recherche d'aide comme un processus décisionnel n'implique pas nécessairement qu'elle est l'aboutissement d'une analyse basée sur une rationalité absolue; ce processus décisionnel est orienté par des contraintes cognitives et affectives qui empêchent d'appréhender toutes les données nécessaires à la recherche de la solution optimale; s'engager dans une démarche d'aide comporte plusieurs inconnues. Ce processus s'inscrit donc dans un contexte de rationalité (March et 
Simon, 1979) où les décisions sont conditionnées par le contexte dans lequel vit la personne. Ce contexte donne une couleur à l'information dont elle dispose, il influence ses valeurs, il fournit une base de comparaison pour l'évaluation de sa situation personnelle et il établit les paramètres à partir desquels elle apprécie les solutions alternatives à sa réalité actuelle.

Plusieurs explications peuvent donner un sens à l'attitude des populations difficiles à rejoindre à l'égard des services sociaux. L'adoption d'une perspective interactionniste conduit à une vision où les concepts de résistance et d'hostilité font place à une explication qui reconnaît le caractère "logique " de leur réaction. En tenant compte du contexte d'où elle tire se rationalité, la motivation de la personne à s'engager dans une démarche d'aide n'est plus abordée comme une caractéristique personnelle statique, mais comme la résultante des interactions entre différents systèmes : la personne, son environnement immédiat (famille, réseau, milieu culturel), les services proposés et les institutions sociales dans lesquels ces services s'inscrivent. L'engagement dans une mesure d'aide peut alors être abordé comme l'aboutissement d'une démarche décisionnelle qui prend place dans une dynamique d'interaction entre un individu, avec ses caractéristiques, ses besoins, ses attentes, lesquels sont influencés par son environnement, et une structure de services qui s'inscrit dans des institutions sociales qui en influencent la nature. La figure qui suit présente un modèle, basé sur les concepts de l'approche écologique (Bronfenbrenner, 1979; 1986; Bouchard, 1987), du processus qui entoure l'engagement dans un mesure d'aide. Cet effort de modélisation ne prétend pas traduire la réalité dans toute sa complexité; il propose une simplification de la réalité en se limitant aux éléments qui apparaissent les plus significatifs du point de vue de l'intervention. L'engagement dans une démarche d'aide y est analysé par un raisonnement qui part du contexte dans lequel évolue la personne pour "comprendre en quoi et pourquoi, dans ce [contexte], les comportements [...] sont en fait rationnels " (Crozier et Friedberg, 1977, p. 200). 
Figure 1

Illustration du processus d'engagement dans une démarche d'aide

Macrosystème

(croyances, valeurs, normes, idéologie)

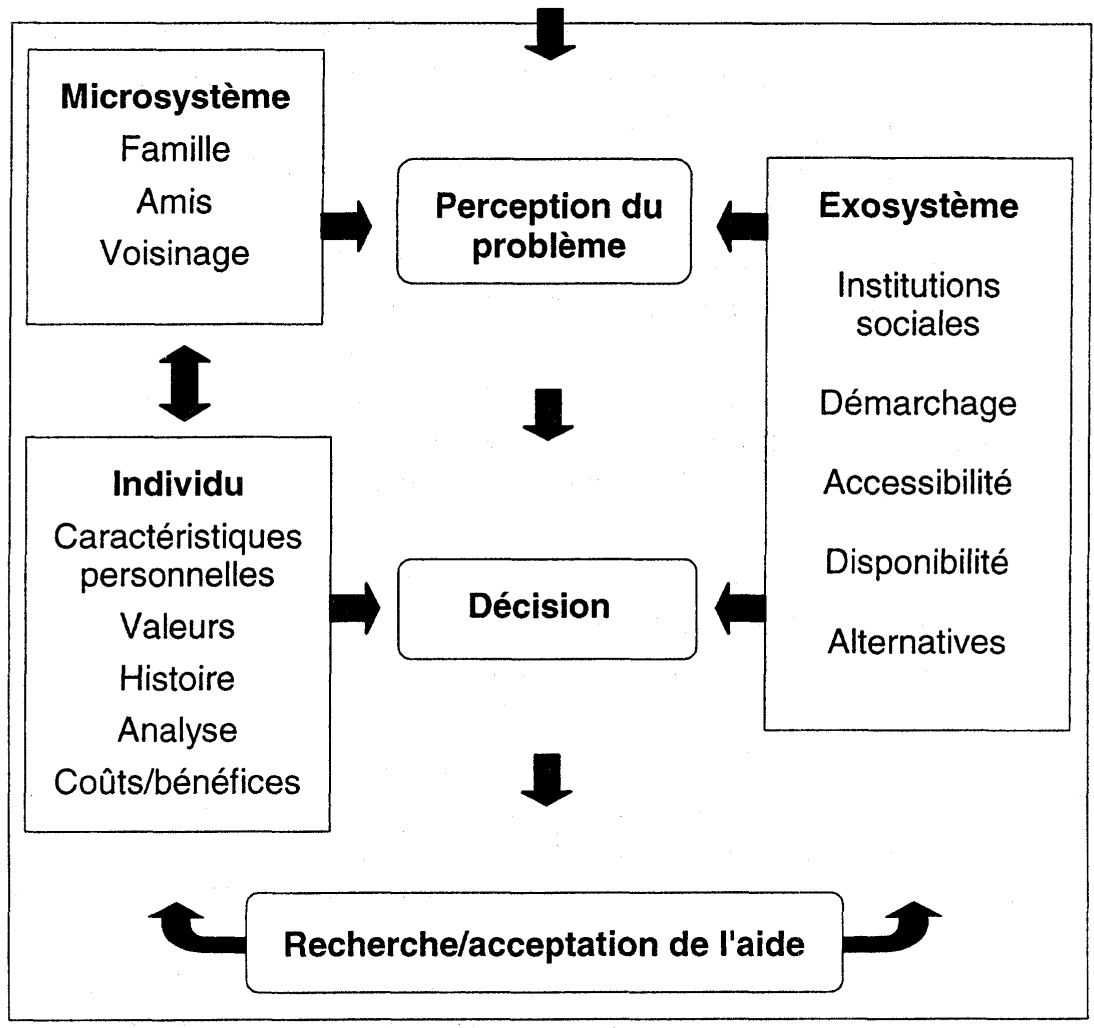

Dans ce modèle, l'intervenant social n'est pas extérieur au processus; comme membre du système d'intervention, il exerce une influence sur la démarche décisionnelle de la personne en difficulté par sa façon d'entrer en contact avec elle et par la nature des stratégies de démarchage qu'il met en place pour la rejoindre. Ces stratégies peuvent prendre différentes formes. Toseland et Rivas (1998) en distinguent quatre : 1) le contact direct, 2) l'information postale, 3) la publicité et 4) la nouvelle. Ces différentes stratégies n'ont évidemment pas toutes la même portée sur le recrutement et, bien que 
le contact direct apparaisse généralement comme la stratégie la plus prometteuse (Toseland et Rivas, 1998), les autres peuvent également déboucher sur des résultats intéressants si l'information qui est transmise rejoint les personnes visées. À cet égard, il est important que l'offre de service soit communiquée dans un langage qui rejoint les personnes à qui elle s'adresse plutôt que dans un style qui correspond à la culture professionnelle de l'intervenant (Robertson, 1989; Heap, 1987; Mullender et Ward, 1991). En plus d'être formulé dans un langage accessible et présenté dans un format attrayant, le message doit également contenir des données sur la nature des services offerts, afin de permettre aux personnes visées de prendre une décision éclairée. C'est pourquoi il est généralement suggéré de fournir des indications sur le but de l'intervention, les endroits, dates, moments, fréquence et durée des activités, de même que sur les frais de participation et les facilités offertes (ex. : service de garderie, transport, matériel didactique). II est également utile de préciser le nom de l'organisme responsable : cette information peut contribuer à donner de la crédibilité au projet.

Dans la planification d'une stratégie de recrutement, un des éléments importants à considérer est la nature de la collaboration des personnes et des organismes qui sont mis à contribution. Si le recrutement implique des partenaires, il est essentiel de s'assurer que les mécanismes de transmission de l'information qui sont utilisés par ces partenaires atteignent leurs objectifs.

\section{QUELQUES ILLUSTRATIONS TIRÉES DES PROJETS DU PACE ${ }^{3}$}

Les projets financés dans le cadre du Programme d'action communautaire pour les enfants (PACE) s'adressent aux enfants et aux familles de milieux défavorisés qui sont considérés comme faisant partie d'une population à risque. Selon les informations recueillies

3. Le Programme d'action communautaire pour les enfants (PACE) est un programme géré par Santé Canada. II fournit une aide financière à des organismes qui souhaitent développer et offrir des services à l'intention des jeunes enfants à risque (0-6 ans) ou de leur famille. En 1995-1996, on comptait, au Québec, 182 projets récurrents financés dans le cadre de ce programme. Un consortium de chercheurs du Centre de recherche sur les services communautaires de l'Université Laval et de l'École nationale d'administration publique ont procédé à l'évaluation de ce programme. Les données présentées dans cet article sont tirées d'un des volets de cette recherche évaluative. 
dans le cadre d'une recherche qualitative portant sur 19 de ces projets, leur démarchage s'articule autour de stratégies ayant toutes pour objectif de faire en sorte que les services offerts soient mieux connus de la population et des intervenants. Cette diffusion d'information dans la communauté est réalisée par trois voies d'action : la publicité, l'information ciblée et l'infiltration.

La publicité consiste essentiellement à faire connaître les nouveaux services rendus disponibles par une information diffusée largement dans la communauté. Cette action passe par l'utilisation de différents canaux : médias (journal local, radio, télévision communautaire), conférence de presse, Publi-Sacs, affiches. Les projets qui recourent à cette stratégie veulent généralement rejoindre une nouvelle clientèle.

L'information ciblée s'adresse plus directement à la clientèle visée. Cette modalité se présente sous différentes facettes selon qu'elle vise la population cible ou les intervenants des organismes du milieu. Dans le premier cas, les moyens qui sont privilégiés sont la distribution de documents d'information et de dépliants, de même que le contact direct. Dans le second, c'est généralement par de l'information verbale ou par lettre que l'information sur le projet est diffusée. Toutefois, même lorsque l'information s'adresse à la population cible, les moyens utilisés exigent la collaboration des organismes de la communauté dans la mesure où c'est par l'intermédiaire de ces organismes que l'information est diffusée. En effet, les dépliants sont généralement distribués dans les présentoirs des organismes et les contacts directs se font très souvent lorsque la population cible utilise les autres services des organismes. Les liens avec les ressources du milieu qui touchent des populations semblables à celles qui sont visées constituent donc des atouts importants pour le recrutement de la clientèle.

La troisième modalité utilisée pour assurer la diffusion du projet pourrait être qualifiée d'infiltration : infiltration parmi la clientèle, d'une part, et infiltration au sein du réseau des organismes, d'autre part. Cette modalité dépasse la diffusion d'information sur le projet et ses activités; elle mise sur l'établissement d'un lien direct entre la communauté et les personnes qui œuvrent dans le projet. En ce sens, elle peut être considérée comme une stratégie de démarchage beaucoup plus proactive. De façon concrète, par rapport à la clientèle 
cible, l'infiltration consiste à aller rejoindre ces personnes là où elles se trouvent, et ce, par différentes stratégies : en étant présent aux endroits qu'elles fréquentent, en étant présent dans les organismes qu'elles consultent, en s'associant à des personnes avec qui elles sont en contact. Comme l'a souligné un intervenant, il ne faut pas hésiter à investir du temps auprès de ces personnes, il faut « perdre du temps " pour établir un véritable contact avec elles. Cette stratégie est particulièrement pertinente pour les organismes qui ne sont pas déjà en lien avec leur clientèle cible et ceux qui tentent de rejoindre une nouvelle clientèle.

Pour ce qui est de l'infiltration au sein du réseau des organismes, l'existence dans une communauté de tables de concertation facilite beaucoup cette opération. En effet, la présence à ces tables devient une occasion privilégiée pour faire connaître son organisme, pour se faire connaître comme personne et pour tisser les liens interpersonnels qui pavent la voie au développement d'un partenariat prometteur.

Dans notre milieu, il y a beaucoup de concertation entre les organismes. [...] Même si on vient juste de "partir " une table de concertation, je pense qu'ily a des choses qui se font encore plus que dans les secteurs qui avaient des organismes plus officiels de concertation. Les organismes en enfance-famille se connaissaient beaucoup, ils se voyaient beaucoup. Ils partagent souvent des clientèles. II n'y a pas d'isolement dans ce sens là. ( $\left.n^{\circ} 2\right)$

Le tableau 2 illustre les différentes stratégies utilisées dans les projets du PACE pour se faire connaître dans la communauté et pour recruter des participants aux activités offertes.

Même si les projets recourent à une diversité de moyens, le recrutement des participants n'en demeure pas moins une difficulté pour plusieurs d'entre eux. Certains motifs d'ordre organisationnel sont avancés pour expliquer les difficultés de recrutement; d'aucuns tiennent aux caractéristiques du projet ou de l'organisme qui en assume la réalisation, d'autres se réfèrent à la contribution des partenaires qui participent au recrutement. 


\section{Tableau 2}

\section{Illustration des différentes modalités de diffusion dans la communauté}

\begin{tabular}{|c|c|}
\hline Stratégie & Extraits d'entrevue \\
\hline \multirow[t]{4}{*}{ Publicité } & $\begin{array}{l}\text { Nous on a fait de la publicité dans les autres organismes } \\
\text { du quartier, dans le journal du quartier et à la radio com- } \\
\left.\text { munautaire. ( } n^{\circ} 4\right)\end{array}$ \\
\hline & $\begin{array}{l}\text { Avec les deux personnes responsables, nous avons en- } \\
\text { trepris de faire une conférence de presse pour annoncer } \\
\text { le projet. On a fait une réception. On a invité les députés. } \\
\left(n^{\circ} 14\right)\end{array}$ \\
\hline & $\begin{array}{l}\text { On m'a dit que c'était super difficile de rejoindre les pa- } \\
\text { rents. C'est là qu'on a pensé envoyer des lettres dans } \\
\text { tous les Publi-Sacs. À partir de là, on a eu un premier } \\
\text { groupe. Après on a encore utilisé les Publi-Sacs et on a } \\
\text { eu un deuxième groupe. (n० 16) }\end{array}$ \\
\hline & $\begin{array}{l}\text { Dans les journaux locaux. II y a aussi un animateur de } \\
\text { radio qui rappelle aux gens, à notre demande, qu'un atelier } \\
\text { va débuter bientôt et qu'il est encore temps de s'inscrire. } \\
\text { (n' 18) }\end{array}$ \\
\hline \multirow{3}{*}{$\begin{array}{l}\text { Information } \\
\text { visant la } \\
\text { population } \\
\text { cible }\end{array}$} & $\begin{array}{l}\text { On s'est concentré pour créer un dépliant. Ensuite, on a } \\
\text { essayé de voir qui seraient les autres organismes qui } \\
\text { pourraient nous référer de la clientèle. (n० 18) }\end{array}$ \\
\hline & $\begin{array}{l}\text { Je demande d'avoir un kiosque à la remise des bulletins. } \\
\text { Ensuite, je fais un article dans le journal de l'école. (n० 16) }\end{array}$ \\
\hline & $\begin{array}{l}\text { On va dans les écoles au début d'année pour présenter } \\
\text { l'organisme. On est dans la cour d'école et on rencontre } \\
\left.\text { les parents. ( } n^{\circ} 11\right)\end{array}$ \\
\hline \multirow{3}{*}{$\begin{array}{l}\text { Information } \\
\text { ciblée } \\
\text { visant les } \\
\text { intervenants }\end{array}$} & $\begin{array}{l}\text { J'ai demandé aux intervenants de faire le tour des CLSC, } \\
\text { au moins de leur téléphoner, pour les informer du projet. }\end{array}$ \\
\hline & $\begin{array}{l}\text { [...] II y a eu aussi des lettres aux organismes commu- } \\
\text { nautaires. }\left(n^{\circ} 15\right)\end{array}$ \\
\hline & $\begin{array}{l}\text { On a rencontré des intervenants de CLSC qui vont dans } \\
\text { des cliniques de vaccination et qui nous disaient que peut- } \\
\text { être qu'ils pouvaient rejoindre des parents qui ont des } \\
\left.\text { difficultés et qui pourraient participer. (n' } n^{\circ} 11\right)\end{array}$ \\
\hline
\end{tabular}




\section{Tableau 2 (suite)}

Infiltration au L'an dernier, on a fait du théâtre dans les ruelles du quarsein de la tier. [...] C'était pour rejoindre les gens qui ne venaient population pas à l'organisme. [...] Ça rendait les gens curieux et les cible jeunes s'impliquaient. Cela a permis à l'organisme d'être encore plus dans le quartier, près des gens. ( $\left.n^{\circ} 11\right)$

J'allais dans la clinique de vaccination et je restais un peu dans la salle d'attente avec les parents pour donner des dépliants. $\left(n^{\circ} 16\right)$

Avant de commencer ma première visite dans le milieu, [...] je suis allée dans un organisme et j'ai demandé si je pouvais aider, comme ça, bénévolement, sur mes heures de travail. [...] Les gens me voyaient les aider à transporter des sacs, les aider à choisir telle grandeur, ramasserdes supports, le même rôle que les gens qui travaillent dans ce comptoir vestimentaire. [...] Ce fut une bonne affaire. $\left(n^{\circ} 5\right)$

Infiltration au Moi, au début de l'année, je rencontre toujours les direcsein des organismes trices d'école. [...] Après, je rencontre tous les professeurs pour leur expliquer le projet. ( $\left.n^{\circ} 17\right)$

On est allé voir toutes les écoles primaires et toutes les garderies du territoire. [...] II y a le bouche à oreille qui joue lorsqu'on se fait connaître. À chaque fois qu'il y avait une petite promotion à faire, on partait et on allait promouvoir nos activités. ( $\left.n^{\circ} 10\right)$

En ce qui a trait aux caractéristiques de l'organisme ou du projet, les motifs mentionnés font référence à des éléments comme le manque de crédibilité dans la communauté, la faiblesse des stratégies de recrutement, le manque d'attrait des activités et l'inadéquation de la période de réalisation des activités. Au regard de la contribution des partenaires, différents facteurs apparaissent sous-jacents à leur collaboration mitigée : méconnaissance du projet, négligence à faire des références de façon systématique, tension entre les organismes, concurrence en matière de services. Le tableau 3 (p. 117) illustre les principaux motifs d'ordre organisationnel mentionnés en explication aux difficultés de recrutement. 
Si certains facteurs d'ordre organisationnel font obstacle au recrutement, d'autres, par ailleurs, y apparaissent favorables. La renommée de l'organisme et la nature des liens qu'il entretient avec les autres organismes de la communauté sont au nombre de ces facteurs facilitants. En effet, l'enracinement de l'organisme, son rayonnement, l'ouverture des partenaires et lintérêt de la communauté sont autant d'éléments qui sont mentionnés comme facilitants pour le recrutement. À l'opposé, lorsqu'il s'agit d'un nouvel organisme ou d'un organisme qui est à redéfinir sa mission, il doit consacrer plus d'énergie à " bâtir ses liens de confiance avec le milieu ». Ces organismes moins bien connus dans leur communauté ont avantage à adopter des stratégies proactives afin de rejoindre leur clientèle cible là où elle se trouve et d'établir des liens avec des partenaires éventuels. Cette approche exige efforts et originalité, mais le jeu en vaut généralement la chandelle. En effet, lorsqu'un bon contact est établi avec les partenaires de la communauté, ces partenaires peuvent se révéler des sources de recrutement très précieuses. Voici quelques exemples d'actions qui, dans les projets du PACE, ont eu des répercussions positives sur le recrutement: faire du porte-àporte dans le quartier pour informer les personnes du projet, se rendre dans les ressources fréquentées par la clientèle visée (comptoirs alimentaires et vestimentaires), offrir des services concrets.

Au niveau du recrutement, c'est sûr qu'il faut être assidu, puis il faut aller les rencontrer dans leur milieu. [...] II faut aller dans le milieu, il faut aller frapper à la porte, c'est comme ça que ça fonctionne, dans le milieu des HLM là, aller leur serrer la main, après ça va bien. $\left(n^{\circ} 7\right)$

Quand on arrive dans une nouvelle communauté, on n'est pas de là, on n'est pas connu. On va être drôlement perçu par la population si on arrive comme ça avec un programme. II faut comme une personne intermédiaire très connue dans le milieu qui nous introduit. [...] De s'associer à un groupe communautaire, c'est un élément qui est facilitant. $\left(n^{\circ} 3\right)$ 


\section{Tableau 3 \\ Les motifs organisationnels liés aux difficultés de recrutement de la clientèle}

\section{Caractéristiques du projet ou de \\ l'organisme}

- Manque de crédibilité dans la communauté

- Faiblesse des stratégies de recrutement

- Nature des activités offertes

- Inadéquation de la période de début des activités
C'est pas sûr que tu vas embarquer dans quelque chose que tu ne connais pas. [...] Ils attendent que certains participent pour avoir leurs impressions [...] Comme on n'avait pas de lien de confiance bâti avec le milieu comme on dit " vulnérable ", j'avais à bâtir ça. $\left(n^{\circ} 2\right)$

Je souhaiterais que le programme ait davantage de crédibilité mais ça va se gagner à la longue. [...] Le programme n'est pas assez connu. Les organismes communautaires, en particulier, réfèrent encore davantage au CLSC qui est une ressource implantée depuis plus longtemps que nous. ( $\left.n^{\circ} 19\right)$

Je trouve que dans le projet il n'y a pas eu assez de temps consacré au recrutement. On n'a pas eu assez de temps. ( $\left.n^{\circ} 18\right)$

On avait prévu 8 rencontres de 3 heures. On s'est dit que c'était peut-être un facteur qui faisait que les parents s'inscrivaient pas parce que c'était pas attirant. $\left(n^{\circ} 18\right)$

C'est pas de leur faute, la subvention est entrée à ce moment-là. L'été c'est un temps mort, les clients ont moins de difficultés aussi, les enfants jouent dehors. [...] Au début juin, il n'y a pas beaucoup de références qui se font. ( $\left.n^{\circ} 19\right)$

\section{Contribution des} partenaires

- Méconnaissance II y a plusieurs groupes de parents qui se mettent du projet sur pied par différents organismes et il faut que tu sois à la fine pointe pour savoir que tel organisme s'adresse à telle clientèle et tel autre à telle clientèle. Ça peut devenir confus si l'orientation ne se fait pas bien. $\left(n^{\circ} 2\right)$ 


\section{Tableau 3 (suite)}

\section{Contribution des} partenaires (suite)

- Négligence à " référer » de façon systématique

Peut-être qu'il y aurait lieu de regarder au niveau de la fréquence de leur contribution pour qu'ils renouvellent la mémoire à leurs intervenants. $\left(n^{\circ} 18\right)$

- Tension entre les organismes

Ily a un peu de tension théorique avec la protection de la jeunesse parce que notre projet ne veut pas donner l'information qui va permettre le signalement dans des cas extrêmes. [...] Ils font peut-être moins de références dû à cela. ( $\left.n^{\circ} 19\right)$

- Concurrence

Les intervenants hésitent à référer des clients de peur de manquer de travail et de risquer ainsi de perdre leur travail. $\left(n^{\circ} 15\right)$

- Surcharge de Dernièrement, ils mont dit qu'ils manquaient de travail clientes. On va essayer d'en envoyer, mais nous autres, on a une liste d'attente effrayante. On n'a pas le temps; ça a bloqué de notre bord. S'ils n'ont pas de références, c'est pas vraiment de leur faute. On a eu ici des changements de poste, des congés de maternité, etc. $\left(n^{\circ} 19\right)$

\section{Le contact pré-groupe}

Si le recrutement permet que certaines personnes se montrent intéressées, ou à tout le moins réceptives, à une offre de service, cette ouverture doit déboucher rapidement sur un engagement plus marqué. En effet, il faut faire en sorte que les personnes qui expriment de l'intérêt en viennent à participer aux activités qui sont proposées. Ce passage, de l'intérêt à l'engagement, se réalise généralement lors du contact pré-groupe. II est souhaitable que ce contact, qui peut se concrétiser dans le cadre d'une entrevue individuelle, d'une rencontre pré-groupe ou d'un appel téléphonique, soit réalisé auprès de tous les participants potentiels à un groupe. L'intervenant peut alors évaluer leur désir et leur capacité de se joindre au groupe, clarifier les buts de l'intervention, établir des liens entre les besoins de chacune des personnes et présenter sommairement le plan d'action (Henry, 1992). Le contact pré-groupe constitue une occasion privilégiée d'identifier les besoins spécifiques de la personne et de vérifier dans quelle mesure l'action envisagée représente une façon adéquate de répondre à ses besoins. 
Pour Manor (1986; 1988), le contact pré-groupe peut exercer une grande influence sur la réussite d'un groupe, puisque la façon dont l'intervenant composera avec l'ambivalence de la personne sera souvent déterminante sur sa décision de joindre ou non le groupe. Ainsi, pour conduire à un engagement réel de la personne, le contact pré-groupe doit lui permettre de voir en quoi ses besoins " personnels » peuvent s'arrimer aux besoins de personnes qui vivent une situation semblable à la sienne.

Toseland et Rivas (1998) attribuent trois fonctions principales au contact pré-groupe : 1) l'explication du but du groupe, 2) la présentation de son fonctionnement et 3 ) la sélection des membres. En ce qui regarde le but, le contact pré-groupe offre la possibilité à l'intervenant d'échanger avec les membres potentiels sur ce que le groupe peut leur offrir. Si cet exercice peut les aider à voir plus clairement les bénéfices que peut procurer la participation au groupe, il est cependant important que l'intervenant évite la persuasion à tout prix, puisqu'une telle attitude produit généralement des effets contraires à ce qui est recherché (Heap, 1987). En fait, la présentation du but du groupe devrait se faire en ayant en tête deux préoccupations : 1) transmettre dans un langage clair, concret et accessible sa conception des buts et du contenu du groupe et 2) éviter de se laisser guider par son désir de vendre le groupe; il faut susciter la motivation plutôt que de recourir à la persuasion.

Au-delà du but visé, il est nécessaire d'informer les membres potentiels des normes qui vont régir le fonctionnement du groupe et du rôle qu'assumera l'intervenant. Cette information contribue généralement à sécuriser les personnes plus craintives devant le groupe (Shulman, 1988). Au moment de la présentation du fonctionnement du groupe, il peut être avantageux de considérer les suggestions suivantes : 1) spécifier clairement ce qui sera attendu des participants (par exemple : être actif, être présent, exprimer son point de vue), 2) reconnaître et explorer les sentiments qui sous-tendent la résistance, 3) mettre en évidence les possibilités qu'offre le groupe et 4) mentionner l'apport positif que chaque membre peut fournir aux autres membres et à l'ensemble du groupe.

En ce qui a trait à la sélection des membres, le contact prégroupe offre à l'intervenant la possibilité de s'assurer qu'il y a correspondance entre les besoins individuels des personnes et le but du 
groupe. Dans cet exercice de sélection, il doit également s'assurer que les personnes ont le désir et sont capables d'entrer en communication avec les autres, et qu'ils peuvent accepter des opinions et des comportements différents des leurs; les personnes imperméables aux autres risquent de demeurer isolées et de freiner la dynamique du groupe (Toseland et Rivas, 1998). Généralement, les groupes efficaces sont des groupes dans lesquels les membres sont interactifs, compatibles et réactifs (Bertcher et Maple, 1977). Les membres sont interactifs lorsqu'ils parlent entre eux et échangent leurs idées et leurs points de vue; ils sont compatibles lorsqu'ils partagent un intérêt commun et qu'ils démontrent un souci des autres; enfin, ils sont réactifs lorsqu'ils sont intéressés au groupe et actifs dans la dynamique d'aide mutuelle.

Plusieurs projets du PACE ont recours à une procédure d'accueil qui vise des objectifs analogues au contact pré-groupe. Comme lillustrent les exemples qui suivent, cette procédure peut prendre diverses formes : contact téléphonique, visite à domicile, entrevue individuelle dans les locaux de l'organisme, rencontre de groupe.

En général, je téléphone et on jase. Des fois, c'est une demiheure et ça peut aller jusqu'à une heure. Quand elles se sentent en confiance, elles se mettent à nous raconter comment elles se sentent, quelles sont leurs difficultés. Quand je vois que le lien de confiance est là [...] je leur dis que ça [le groupe] existe et que ça peut leur apporter de quoi. Mais je ne force pas. ( $\left.n^{\circ} 2\right)$

Je rencontre individuellement chaque participante et, avec un questionnaire, je fais le tour de la situation familiale, financière, qu'est-ce qu'elles aimeraient venir chercher. [...] Ça me permet de voir au niveau des besoins. $\left(n^{\circ} 8\right)$

Dans le premier atelier, on fait un tour de table, on leur demande d'écrire leur nom sur un carton, ce qu'ils font dans la vie et le nom de leur enfant. [...] Comme c'est un groupe qui vise une certaine ouverture, je veux que le groupe soit à l'aise assez rapidement. Ils me disent ça commence raide, mais ils disent que c'est la meilleure chose que tu as faite. ( $\left.n^{\circ} 16\right)$

L'accueil peut remplir plusieurs fonctions, comme établir les bases d'un climat de confiance, clarifier les attentes du participant, cerner sa motivation, fournir des informations sur le projet et ses différents volets d'activités, préciser les conditions de participation, obtenir des informations plus précises sur le participant. La qualité 
de l'accueil influence l'engagement et, en ce sens, elle peut enrichir ou éroder les efforts consacrés au moment du recrutement.

On a parlé près d'une heure au téléphone. Moi, je trouve que ça a "fitté " tout de suite. [...] Je veux dire que j'ai été comprise, je ne me sentais ni jugée ni tous ces, comment on dit ça, les préjugés. ( $\left.n^{\circ} 2\right)$

Donc, si le recrutement auprès des populations difficiles à rejoindre gagne en efficacité lorsque les stratégies utilisées impliquent une alliance entre différents organismes d'une communauté, il y a également un intérêt certain à mettre en place des procédures d'accueil ou de contact pré-groupe afin de maintenir et de stimuler l'intérêt éveillé chez les participants potentiels par les stratégies de démarchage.

\section{CONCLUSION}

L'intervention auprès des populations défavorisées pose un défi de taille pour les travailleurs sociaux. Si, pour certains, ces populations sont “ déprimées et déprimantes " (Platteau, 1994), elles n'en sont pas moins porteuses de ressources et de compétences, et elles ont surtout des besoins criants qu'elles ne sont pas en mesure de satisfaire. Les intervenants sociaux doivent se donner des moyens pour mieux comprendre la réalité de ces personnes et répondre plus adéquatement à leurs besoins.

Un premier obstacle à franchir dans l'intervention auprès des personnes défavorisées tient à leur méfiance à l'égard des services sociaux. Or, sur ce plan, il faut éviter le piège d'en faire les seules responsables de cette situation; plusieurs facteurs entrent en jeu dans la décision d'une personne de s'engager dans une démarche d'aide. La perspective interactionniste présente une avenue intéressante à cet égard en reconnaissant l'influence du contexte dans lequel se trouve une personne et en présumant d'un fondement " logique » à son comportement. En évitant d'expliquer l'attitude à l'endroit des services sociaux en considérant les seules caractéristiques personnelles, cette perspective soulève l'importance de s'intéresser aux systèmes de distribution de services et aux liens qui existent entre les organismes. 
Les données tirées de la recherche sur les projets du PACE fournissent des indications intéressantes sur le rôle des organismes dans l'action auprès des populations défavorisées. En effet, les commentaires recueillis dans le cadre de cette recherche indiquent que pour les intervenants, si les difficultés à rejoindre les populations défavorisées tiennent pour une bonne part à des problèmes intra et inter-organisationnels, en contrepartie c'est dans des actions énergiques, directement auprès des personnes visées et en collaboration avec les partenaires de la communauté que se trouvent les avenues de solution. Ces observations rejoignent en bonne partie les conclusions de l'étude de Turcotte et Paquet (1996) quant à l'importance 1) de bien connaître la problématique et les besoins de la population cible, 2) de privilégier le contact direct lors du démarchage, 3 ) de fournir des informations précises et claires sur le groupe, et 4) de proposer une intervention qui répond aux besoins de façon stimulante et originale.

Les résultats obtenus par les projets du PACE indiquent qu'il est possible d'agir auprès des populations difficiles à rejoindre, même si la tâche n'est pas facile. La créativité, la ténacité, la conviction et l'ouverture sont des caractéristiques qui marquent l'action quotidienne de ces intervenants. Mais, au-delà de ces attitudes, c'est principalement à travers des actions concertées, réalisées en alliance avec les ressources de la communauté et dans le respect des sensibilités des personnes défavorisées que se trouvent les voies les plus prometteuses pour venir en aide aux populations dites difficiles à rejoindre.

Daniel TURCOTTE

Professeur

École de service social Université Laval 


\section{Références bibliographiques}

ANTONUCCI, T.C. et J.S. JACKSON (1990). " The role of reciprocity in social support ", dans B.R. Saranson, I.G. Saranson et G.R. Pierce (dir.). Social support : an interactional view, New York, John Wiley \& Sons, p. 173-198.

ASSER, E.S. (1978). " Social class and help-seeking behavior ", American Journal of Community Psychology, 6, p. 465-494.

BEHROOZI, C. (1992a). "A model for social work with involuntary applicants in groups ", Social Work with Groups, vol. 15, nos 2-3, p. 223-238.

BEHROOZI, C.S. (1992b). « Groupwork with involuntary clients : remotivating strategies, Groupwork, vol. 5, n², p. 31-41.

BertCHER, H. et F. MAPLE (1977). Creating Groups, Newbury Park, CA, Sage.

BILOdEAU, A. (1994). Dynamique de la recherche d'aide des femmes victimes de violence conjugale ", dans M. Rinfret-Raynor et S. Cantin (dir.), Violence conjugale, Recherches sur la violence faite aux femmes en milieu conjugal, Montréal, Gaëtan Morin Éditeur, 513 p.

BOUCHARD, C. (1987). "Intervenir à partir de l'approche écologique : au centre, l'intervenante ", Service social, vol. 36, nos 2-3, p. 454-478.

BRETON, M. (1991). Ouvrir la pratique du service social de groupe sur la communauté : vers un modèle de partenariat, Faculté de service social, Université de Toronto, texte inédit.

BRONFENBRENNER, U. (1979). The experimental ecology of human development, Cambridge, Mass., Harvard University Press.

BRONFENBRENNER, U. (1986). " Ecology of the family as a context for human development : research perspectives ", Developmental Psychology, vol. 22, no 6, p. 723-742.

CANTIN, S. (1998). " Quinze offres d'aide pour une demande », Intervention, no 106, p. 35-55.

CRESPO-MEDINA, J.D. (1988). Help-seeking behavior and its social context : social networks, network orientation and social acceptability of the problem, Boston, Boston University, Thèse de doctorat.

Crozier, M. et E. Friedberg (1977). L'acteur et le système, Paris, Seuil.

DULAC, G. (1997). Les demandes d'aide des hommes, Montréal, Université McGill, Centre d'études appliquées sur la famille. 
FISHER, E.H., D. WINER et S.I. ABRAMOWITZ (1983). " Seeking professional help for psychological problems ", dans A. Nadler, J.D. Fisher et B.M. DePaulo (dir.), New Directions in Helping. Applied perspectives on help-seeking and receiving, New York, Academic Press, p. 163-185.

FORTIN, S. et G. LEGAULT (1994). « Mode de résolution de problèmes des jeunes familles immigrantes : résultats de recherche ", Service social, vol. 43, n०2, p. 97-128.

GooD, G.E., D.M. DELL et L.B. MINTZ (1989). “ Male role and gender role conflict : relations to help seeking in men ", Journal of Counseling Psychology, vol. 36, n 3, p. 295-300.

GREenBERG, M.S. (1980). "A theory of indebtedness ", dans K. Gergen, M.S. Greenberg et R.H. Willis (dir.), Social exchange : Advances in Theory and Research, New York, Plenum Press.

GREENBERG, M.S. et D.R. WESCOTT (1983). “ Indebtedness as a mediator of reactions to aid ", dans J.D. Fisher, A. Nadler et B.M. DePaulo (dir.), New Directions in Helping : Recipient Reactions to Aid, New York, Academic Press, p. 85-112.

Gross, A.E. et P.A. McMULLEN (1983). “ Models of help-seeking process ", dans B.M. DePaulo, A. Nadler et J.D. Fisher (dir.), New Directions in Helping. Help-seeking, New York, Academic Press, p. 45-70.

HEAP, K. (1987). La pratique de service social avec les groupes, Paris, Éditions ESF.

HenRY, S. (1992). Group Skills in Social Work, Pacific Grove, CA, Brooks/ Cole.

HiRAYAma, H. et K. HiRAYAma (1986). " Empowerment through group participation : process and goal ", dans M. Parnes (dir.), Innovations in social group work : feedback from practice to theory, New York, Haworth Press, p. 119-131.

JOHNSON, M.E. (1988). " Influences of gender and sex role orientation on help-seeking attitudes ", The Journal of Psychology, vol. 122, n 3, p. 237-241.

MANOR, O. (1986). " The preliminary interview in social groupwork : finding the spiral steps ", Social Work with Groups, vol. 9, n² 2, p. 21-39.

MANOR, O. (1988). "Preparing the client for social groupwork : an illustrated framework ", Groupwork, vol. 1, n², p. 100-114.

MARCH, J.G. et H.A. SIMON (1979). Les organisations, Paris, Dunod.

MULLENDER, A. et D. WARD (1991). Self-directed groupwork. Users take action for empowerment, Londres, Whiting \& Birch. 
NADLER, A. (1983). "Personal characteristics and help-seeking ", dans B.M. DePaulo, A. Nadler et J.D. Fisher (dir.), New Directions in Helping. Help-seeking, New York, Academic Press, p. 303-340.

Platteau, G. (1994). "Approche des familles défavorisées ", dans O. Amiguet et C. Julier (dir.), Travail social et systémique, Genève, Les Éditions IES, p. 151-184.

ROBERTSON, J.M. (1989). Men who avoid counseling : identifying personality correlates and preferences for assistance alternatives, Thèse de doctorat, Santa Barbara, University of California.

ROGLER, L.H. et D.E. CORTES (1993). Help-seeking pathways : a unifying concept in mental health care, American Journal of Psychiatry, 150, p. 554-561.

ROONEY, R.H. (1988). "Socialization strategies for involuntary clients ", Social Casework, vol. 69, n 3, p. 131-140,

ROONEY, R.H. (1992). Strategies for Work with Involuntary Clients, New York, Columbia University Press.

SHAPIRO, G. (1984). « Help seeking : why people don't ", Research in the Sociology of Organizations, 3, p. 213-236.

SHULMAN, L. (1988). " Groupwork practice with hard-to-reach clients : a modality of choice ", Groupwork, 1, p. 5-16.

SHULMAN, L. (1992). The Skills of Helping, Itasca, III., F.E. Peacock.

TIJHUIS, M.A.R., L. PETERS et M. Foets (1990). «An orientation toward help-seeking for emotional problems ", Social Science and Medicine, vol. 31, n० 9, p. 989-995.

TOSELAND, R.W. et R.F. Rivas (1998). An introduction to group work practice ( $3^{\ominus}$ éd.), Toronto, Allyn and Bacon.

Tousignant, M. (1987). L'étiologie sociale en santé mentale, Montréal, UQAM, Laboratoire de recherche en écologie humaine et sociale.

TURCOTTE, D., D. DAMANT et J. LINDSAY (1995). " Pour une compréhension de la démarche de recherche d'aide des conjoints violents ", Service social, vol. 44, n², p. 91-110.

TURCOTTE, D. et J. PAQUET (1996). Le recrutement en service social des groupes, Cahiers du service social des groupes, no 12, Québec, Université Laval, École de service social.

Turcotte, D., C. SAMson, G. Lessard et A. BeAudoin (1997). De l'intention à l'évaluation : la dynamique de réalisation des projets d'action communautaire - Évaluation des projets du PACE au Québec (vol. 2), Québec, Université Laval, Centre de recherche sur les services communautaires. 
VAUX, A. (1988). Social support : theory, research, and intervention, New York, Praeger.

VEROFF, J., E. DOUVAN et R. KULKA (1981). The Inner America, New York, Basic Books.

WILLIAMS, K.B. et K.C. WILLIAMS (1983). "A social impact perspective on the social inhibition of help-seeking ", dans B.M. DePaulo, A. Nadler et J.D. Fisher (dir.), New directions in helping. Help-seeking, New York, Academic Press, p. 187-204.

YING, Y.W. et L.S. MILLER (1992). “ Help-seeking behavior and attitude of Chinese Americans regarding psychological problems ", American Journal of Community Psychology, vol. 20, n 4, p. 549-556. 